\title{
Die Gesteinsabhängigkeit von Solifluktionsformen in der Ostschweiz und in den Anden Perus und Boliviens
}

\author{
Kurt Graf \\ Geographisches Institut der Universität Zürich
}

Solifluktionsformen entstehen im Zusammenwirken vieler Faktoren, wie Klima, Höhen- und Breitenlage, Lokallage (insbesondere Exposition und Hangneigung), Vegetation und Gesteinsunterlage. Dabei kann jeder dieser Einflüsse als Minimalfaktor auftreten und im Extremfall die Bildung von Strukturböden verhindern. Eine gewisse Ausnahme stellt das Gestein dar, dessen Wirkung außerordentlich vielfältig ist. Die folgenden Betrachtungen basieren auf Untersuchungen im Schweizerischen $\mathrm{Na}$ tionalpark (Unterengadin) und in den peruanischen und bolivianischen Anden.

Wie Elsasser (1968) gezeigt hat, treten Solifluktionsformen auf verschiedenstem Gestein auf. Östlich von Lima, im zentralen Teil der Westkordillere bei Ticlio ( $77^{\circ} 13^{\prime}$ WL, $\left.11^{\circ} 36^{\prime} \mathrm{SB}\right)$ fand ich seine Aussagen bestätigt für Sandstein, Kalk- und Tonschiefer, Andesit, Quarzit und Basalt. Zudem existieren
Solifluktionsformen häufig auf Intrusivgesteinen, wie Diorit, Quarzmonzonit, seltener auf Andesitparphyr und vulkanischem Tuff 1 . In der Cordillera Real nördlich La Paz finden sich noch zusätzlich Strukturböden auf leicht metamorphem Ton (Formation Pampa nach Jauregui, 1969) und rotvioletter, feinkörniger Grauwacke (F. R. Koeberlin, zit. nach Jauregui, 1969). Vermutlich können sich auf jedem Material Solifluktionsformen ausbilden. In meinen Arbeitsgebieten habe ich jedenfalls noch kein Gestein angetroffen, das ihre Bildung vollständig verunmöglicht. Ihre Häufigkeit schwankt allerdings stark, je nach Gestein. Auf die ungleiche Eignung des Gesteinsmaterials weist schon Höllermann (1967, S. 153) hin. Eine Erklärung liegt darin, daß das Gestein andere Solifluktionsfaktoren stark beeinflußt und so indirekt über Existenz, Art und Ausbildungsgrad von Solifluktionsformen entscheidet.

\begin{tabular}{|c|c|c|c|}
\hline \multirow[b]{2}{*}{ bewirkt z. B. } & \multicolumn{2}{|r|}{ Einfluß der Gesteine auf } & \\
\hline & $\begin{array}{l}\text { Relief } \\
\text { schroffe Granitfels- } \\
\text { wände }\end{array}$ & $\begin{array}{l}\text { Vegetation } \\
\text { aufgelockerte, karge } \\
\text { Vegetationsdecke }\end{array}$ & $\begin{array}{l}\text { Lokalklima } \\
\text { gute Wärmeleitung mit } \\
\text { stark zerkleinertem Schutt }\end{array}$ \\
\hline und entscheidet über & $\begin{array}{l}\text { Existenz von Struktur- } \\
\text { böden }\end{array}$ & $\begin{array}{l}\text { Formen der gebundenen } \\
\text { oder ungebundenen } \\
\text { Solifluktion }\end{array}$ & $\begin{array}{l}\text { Kleinformen, Groß- } \\
\text { formen oder überhaupt } \\
\text { keine }\end{array}$ \\
\hline
\end{tabular}

Figur 1. Schema zum Gesteinseinfluß auf die Solifluktion

Zwei Beweise sollen zeigen, wie genau man den Gesteinseinfluß erkennen kann:

a) Lokal bestimmt oft das Gestein, welche Solifluktionsform auftritt. Dies wird besonders an petro-

Ort

Fuorcla Champatsch, Schweiz, $2800 \mathrm{~m}$ ü. M. (815.800/191.900) bzw. (10 $\left.{ }^{\circ} 16^{\prime} \mathrm{EL}, 46^{\circ} 51^{\prime} \mathrm{NB}\right)$ westlich Lago Milluni, Bolivien, $4830 \mathrm{~m}$ ü. M. $\left(68^{\circ} 10^{\prime} \mathrm{WL}, 16^{\circ} 20^{\prime} \mathrm{SB}\right)$ südwestlich Santa Clara, Peru, $4850 \mathrm{~m}$ ü. M. $\left(77^{\circ} 9^{\prime} \mathrm{WL}, 11^{\circ} 37^{\prime} \mathrm{SB}\right)$ graphischen Grenzen deutlich, wo Solifluktionsformen plötzlich wechseln können (Abb. 1). Die folgenden Beispiele mögen diesen Wechsel verdeutlichen.

Solifluktionsformen
Erdstreifchen
Steinstreifen
Erdstreifchen
Girlanden
Erdstreifchen
Zellenboden und
Erdknospen (Abb. 1)

Solifluktionsformen

Serpentin

Bündnerschiefer

Chirquini-Schiefer

Sandstein

grauer Kalk

rötlicher, härterer Kalk
Erdstreifchen
Steinstreifen
Erdstreifchen
Girlanden
Erdstreifchen
Zellenboden und
Erdknospen (Abb. 1)

Figur 2. Beispiele zum Wechsel der Solifluktionsformen bei petrographischen Grenzen 


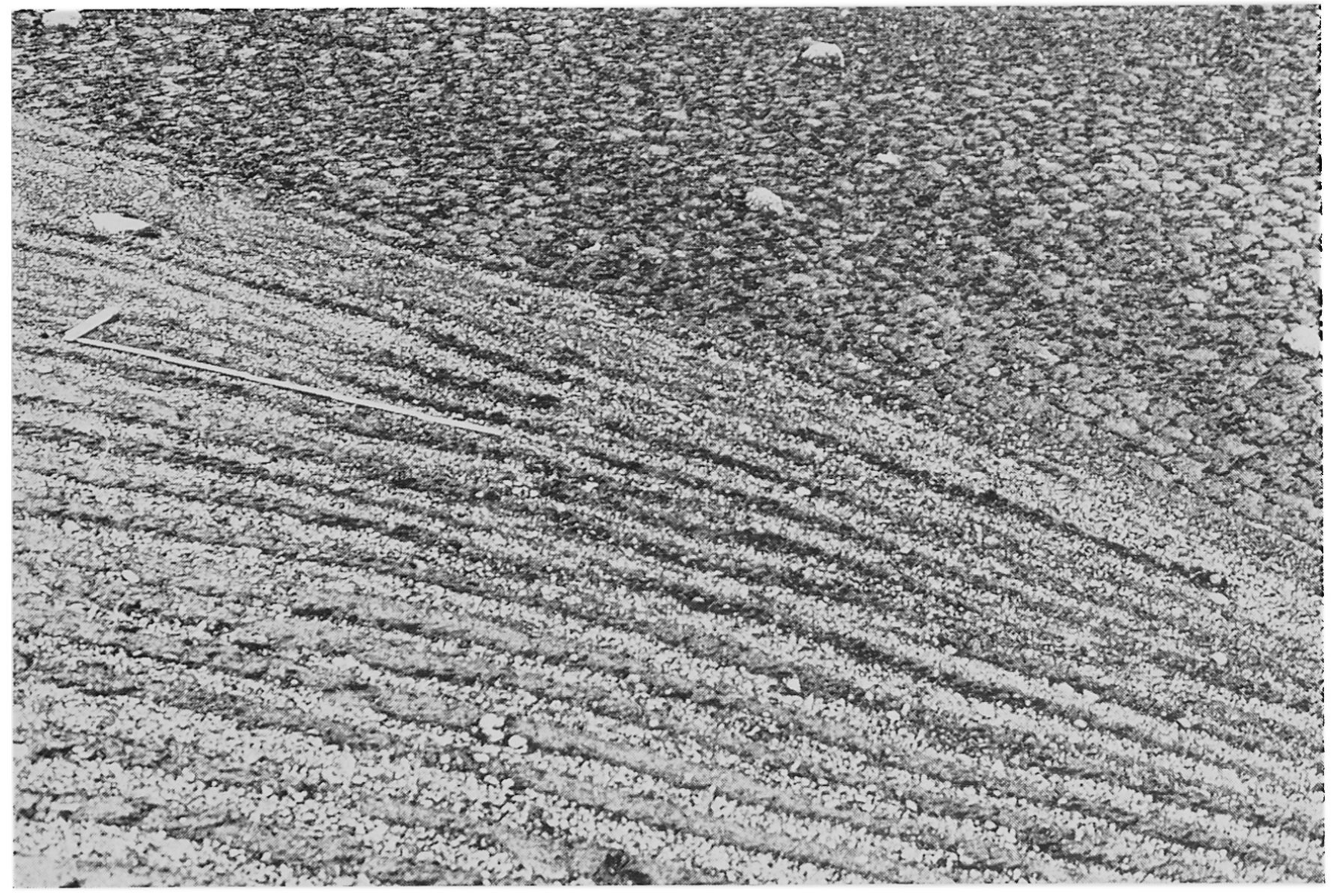

Abbildung 1. Gesteinsgrenze südwestlich Santa Clara, $4850 \mathrm{~m}$ ü. M., (77 9' WL, $11^{\circ} 37^{\prime} \mathrm{SB}$ ). Vorne sind Erdstreifen auf grauem Kalk, hinten Zellenböden auf rötlichem Kalk

Oft fand ich hingegen ausgedehnte Hänge, die ungeachtet der Wechsel von Kalk zu Sandstein, Quarzit oder Schiefer voller Erdstreifchen waren. Eine Gesteinsgrenze bewirkt nicht unbedingt einen Wechsel der Solifluktionsform. Es ist lediglich festzuhalten, daß jedes Material die Solifluktion spezifisch beeinflußt.

b) Großräumiger kann man den Gesteinseinfluß untersucihen, wenn man in einem Gebiet Solifluktiansformen flächenhaft kartiert und ihren Untergrund bestimmt. Im Idealfall treten einige Gesteinsgruppen im Arbeitsgebiet gleich häufig auf. Sodann kann man ihr mittleres Verhalten bezüglich der Solifluktion ermitteln. Als Testform greife ich die weit verbreiteten Erdstreifen heraus. Sie zeigen in meinem $330 \mathrm{~km}^{2}$ großen bolivianischen Arbeitsgebiet rund um den Lago Milluni deutlich gestaffelte Vorkommen, je nach Eignung des Gesteins. Auf leicht metamorphen Tonen und Tonschiefern der Formation Pampa finden sich Erdstreifen auf 4550 $-4950 \mathrm{~m}$ ü. M. Anders liegt ihre Höhenverbreitung auf Gesteinen der Formation Chirquini (Muriel, 1967), grobbankigenTonschiefern und hartem Quarzitsandstein. Mit 4650-5100 m ü. M. kommen sie in 100 bis $150 \mathrm{~m}$ höherer Lage vor als auf den feineren Pampa-Schiefern. Weniger verbreitete Gesteine wie die grobblockigen kristallinen Schiefer der «Pizarras Cordillera Real» und die Grauwacke der Formation Zapla tragen Erdstreifen auf 4750-4900 $\mathrm{m}$ ü. M. Höhe. Konsistente, wenig wärmeleitende Gesteine verlangen offenbar ein strenges Klimaregime und bilden Solifluktionsformen erst in entsprechend höherer Lage aus. Einen gleichen Schlu3 lassen auch meine Untersuchungen im Unterengadin zu. Im $400 \mathrm{~km}^{2}$ messenden Arbeitsgebiet liegt häufig Dolomit vor und in kleineren Vorkommen Bündnerschiefer, Granit, Verrucano und Serpentin. Erdstreifen findet man auf Dolomit in Höhen von 2300-2750 $\mathrm{m}$ ü. M. Höhere Verbreitung zeigen sie auf Gesteinen, die für die Solifluktion weniger geeignet sind: auf Granit und Verrucano 2400 $2950 \mathrm{~m}$ ü. M. Auf Serpentin liegen sie sogar auf $2600 / 2650 \mathrm{~m}$ ü. M.-3000 m ü. M., was einer mittleren Höhenverschiebung von $300 \mathrm{~m}$ gegenüber den Formen auf Dolomit entspricht.

Zusammenfassend will ich festhalten, daß dem Gesteinsmaterial für die Ausbildung und die Höhenlage von Solifluktionsformen entscheidende Be- 
deutung zukommt. Das Gestein kann nicht etwa die Bildung von Strukturböden absolut verhindern. Oft aber gibt dieses den letzten Ausschlag, welche bestimmte Solifluktionsform sich ausbildet, in welcher Qualität und Größe und in welcher Höhenlage.

\section{Literatur}

Elsasser H.: Untersuchungen an Strukturböden im Kanton Graubünden. Diss. Univ. Zürich, 1968. Höllermann P. W.: Zur Verbreitung rezenter periglazialer Kleinformen in den Pyrenäen und Ostalpen. Göttinger Geogr. Abh., 1967.

Jauregui Castillo J.: Estudio geológico minero de la región Milluni-Zongo. Diplomarbeit Univ. La Paz 19692.

Kelletat D.: Rezente Periglazialerscheinungen im Schottischen Hochland. Göttinger Geogr. Abh., 1970.

Morococha Ore Inventory, Vol. II der Minengesellschaft Cerro de Pasco Corporation, Lima 1970. Muriel Zabala C.: Estudio geológico y minera- lógico de la región de Milluni. Diplomarbeit Univ. La Paz, 1967.

Urquidi Barrau F.: Estudio geológico de la región Milluni-Unduavi. Diplomarbeit Univ. La Paz 1966.

\section{Anmerkungen}

1 Höllermann (1967) bestätigt, daß vulkanische Lokkergesteine mit hohem Feinmaterialanteil recht gute Fließeigenschaften haben.

2 Den Diplomarbeiten an der Universidad Mayor de San Andrés in La Paz kommt der Rang einer Dissertation zu, da in Bolivien kein Doktortitel erworben werden kann.

\section{Résumé}

Le matériel du sol influence les formes de solifluxion, surtout l'existence d'un certain type et sa qualité. Souvent, des limites pétrographiques effectuent un changement de formes de solifluxion. 